\title{
A microtitre plate assay for characterizing insensitive acetylcholinesterase genotypes of insecticide-resistant insects
}

\author{
Graham D. Moores, Alan L. Devonshire and Ian Denholm \\ Institute of Arable Crops Research, Rothamsted Experimental Station, Harpenden, Herts., AL5 $2 J Q$, \\ UK
}

\begin{abstract}
A rapid technique is described for characterizing and monitoring, in single insects, the insensitivity of acetylcholinesterase (AChE) to organophosphorus and carbamate insecticides. Ninety-six insects are homogenized simultaneously in a microtitre plate and portions (e.g. 0.05 for Musca domestica L.) assayed colorimetrically with acetylthiocholine in the presence and absence of diagnostic concentrations of insecticide. Reactions are monitored by a kinetic microplate reader linked to a microcomputer that determines mean AChE activities automatically by linear regressions of absorbance-time data. Mean inhibited activity is then expressed as a percentage of uninhibited activity. Several inhibitors can be tested against the same insect to yield an 'insensitivity profile' of individuals and strains. In tests on $M$. domestica adults of known AChE genotype, the assay clearly distinguished not only between a sensitive and two slightly (3-15-fold) insensitive $\mathrm{AChE}$ variants but between all six genotypic combinations of these three alleles.
\end{abstract}

\section{Introduction}

Biochemical tests for specific insecticide resistance $(R)$ mechanisms can provide a powerful means of monitoring the selectivity of control treatments and appraising putative tactics for countering resistance (Brown \& Brogdon, 1987; Devonshire, 1987). To be fully effective, however, such techniques must be applicable to large numbers of single insects and be capable of distinguishing between heterozygotes and homozygotes for $\mathrm{R}$ genes.

Work on insect acetylcholinesterase (AChE), the target of organophosphorus and carbamate insecticides, has identified many enzyme variants (Hama, 1983), even within a single species (Devonshire, 1987), with different patterns of insensitivity to compounds of these chemical groups. Assays developed to diagnose these variants in single insects (Oppenoorth et al., 1977; Devonshire \& Moores, 1984a; Raymond et al., 1985; Hemingway et al., 1986) show contrasting limitations; those enabling high throughput can give limited resolution of $\mathrm{AChE}$ genotypes, even though the insensitivity is $>100$-fold (Hama, 1983) or $>1000$-fold (Hemingway et al., 1986), whilst those giving the clearest definition are timeconsuming to perform. We report here on a microtitre plate assay that overcomes both limitations.

\section{Materials and methods}

Strains of Musca domestica $L$.

Cooper (hereafter termed ' $S$ ')-a susceptible reference strain (Farnham, 1973) homozygous for an $\mathrm{AChE}$ variant showing base-line sensitivity to the organophosphates used. 
49R, WeyR - two strains homozygous for AChE variants with contrasting patterns of insensitivity to organophosphates. $49 \mathrm{R}$ was derived in 1987 from a multi-resistant Danish strain $\left(49 \mathrm{r}_{2}\right.$ b) as a single-pair cross consistently showing reduced sensitivity (measured by techniques described below) to azamethiphos. At the same time, a similar procedure was used to derive WeyR from the multi-resistant Weymann strain collected from a pig farm in the German Federal Republic. The AChE variant in 49R was probably identical to that in strain arD isolated previously from the same Danish stock (Sawicki, 1974; Devonshire \& Moores, 1984b) but since discarded. We found the AChE insensitivity in WeyR to be indistinguishable from that of strain $\mathrm{CH}_{2}$ (Oppenoorth et al., 1977) isolated and characterized from the tetrachlorvinphos-resistant strain first reported by Tripathi \& O'Brien (1973).

\section{Enzyme assay}

Acetylcholinesterase activity was measured using acetylthiocholine as substrate and detecting the released thiol colorimetrically $\left(\mathrm{A}_{405}\right)$ by its reaction with 5,5'-dithiobis $(2$ nitrobenzoic acid) (DTNB) (Ellman et al., 1961). Enzyme inhibition was determined in the presence of substrate (Devonshire \& Moores, 1984a).

Single house-flies were homogenized in $200 \mu \mathrm{l}$ phosphate/Triton $(0.1 \mathrm{~m}$ phosphate buffer, pH 7.5, containing $1 \%$ Triton $\mathrm{X}-100$ ) in separate wells of microtitre plates (NUNC). All 96 flies in each plate were homogenized simultaneously, using a multihomogenizer designed for flat-bottomed microtitre plates (ffrench-Constant \& Devonshire, 1987), and left at $4^{\circ} \mathrm{C}$ for at least $1 \mathrm{~h}$ to ensure sufficient tissue solubilization. Homogenates were then diluted in another microtitre plate by adding $50 \mu \mathrm{l}$ to a further $200 \mu \mathrm{l}$ phosphate/Triton. Diluted homogenate $(50 \mu 1)$, DTNB $(50 \mu 1)$ and phosphate/Triton $(80 \mu \mathrm{l})$ were equilibrated in new microtitre plates at room temperature, using three replicates per fly to give an uninhibited control synchronized with two inhibition reactions. All dilutions and reactants were dispensed rapidly using eight-channel micropipettes (Finn). Assays were started by adding acetylthiocholine iodide in buffer $(20 \mu \mathrm{l})$, with or without inhibitor, to give substrate and DTNB concentrations of $1 \mathrm{~mm}$ in the final assay volume of $200 \mu \mathrm{l}$. Only 0.05 of a house-fly is used in each well, so that several inhibitors or concentrations of inhibitor can be used to characterize the 'insensitivity profile' of each individual fly. However, only dichlorvos and azamethiphos were needed to distinguish the genotypes examined here; preliminary experiments established their optimal concentrations as 10 and $0 \cdot 2 \mu \mathrm{M}$, respectively, with an assay time of $10 \mathrm{~min}$.

Assays were performed on a $V_{\max }$ kinetic microtitre plate reader (Molecular Devices), utilizing the SOFTmax software run on a Packard-Bell (IBM-type) microcomputer, which takes absorbance readings (at $405 \mathrm{~nm}$ ) automatically as follows: 25 in the first $2 \mathrm{~min}, 15$ between 2 and $5 \mathrm{~min}$, and 10 between 5 and $10 \mathrm{~min}$. The readings were summarized by fitting linear and non-linear regressions to data from each well. Linear regressions were performed by the microcomputer, and non-linear (single- and double-exponential models) were fitted using Maximum Likelihood Program (Ross, 1987) after automated data transfer to a VAX mainframe computer.

\section{Results and discussion}

Preliminary experiments showed that the sex, age, diet (milk-fed $v s$ non milk-fed) and rearing temperature $\left(20\right.$ vs $\left.28^{\circ} \mathrm{C}\right)$ had no discernible effect on the expression or inhibition of AChE activity.

The printout of 96 reaction curves (Fig. 1) and the fitting of linear regressions are standard features of the $V_{\max }$ reader in stand-alone mode. Greater flexibility in data presentation and analysis is possible by interfacing with the SOFTmax program. The occasional 'noise' (e.g. well 11F) caused when using fractions of whole fly homogenates can be eliminated by using only fly heads, where most of the AChE is located. However, the improvement achieved will not justify the extra work this entails when analysing a large number of insects.

The progress of reactions is clearly apparent, those with inhibitors following first order kinetics as in the closely-related technique described previously (Devonshire \& Moores, 


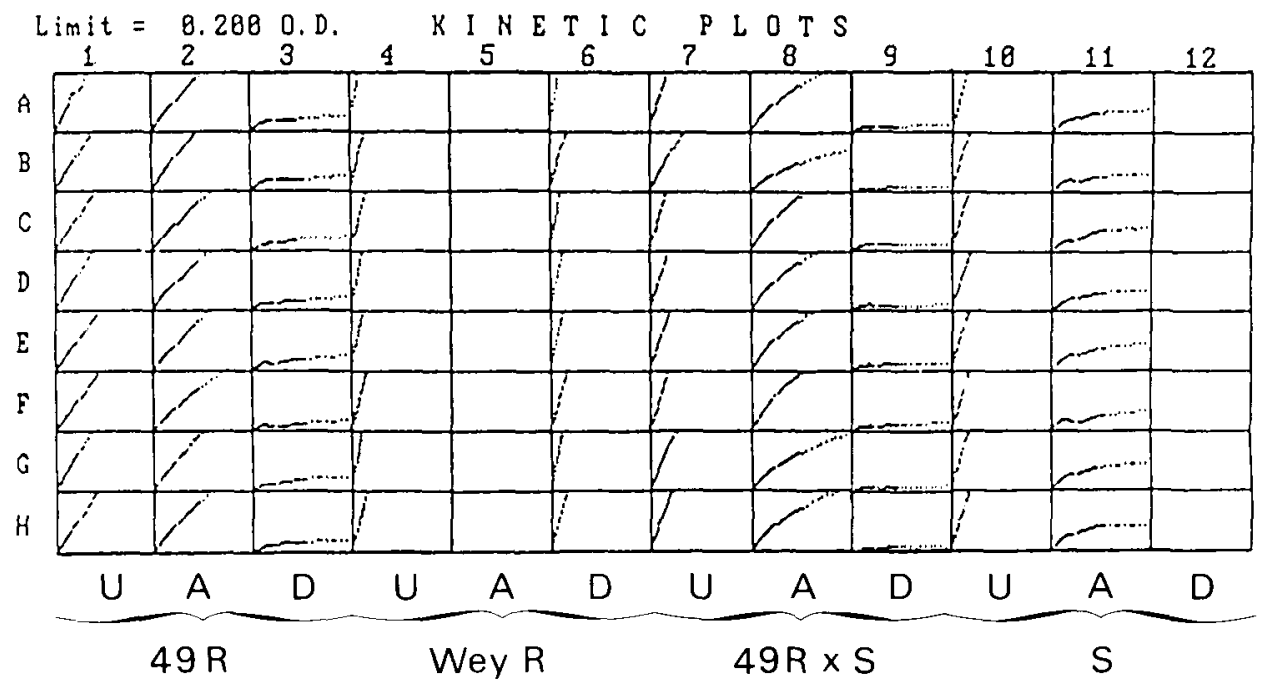

Fig. 1.-Kinetic plots showing changes in absorbance $\left(\mathrm{A}_{405}\right)$ with time for 96 reactions in individual wells of a microtitre plate. Each group of three columns shows data for eight replicate insects (rows A-H) of a strain or cross, assayed without inhibitor (U), and in the presence of $0.2 \mu \mathrm{M}$ azamethiphos (column A) or $10 \mu \mathrm{m}$ dichlorvos (column D). Reactions were monitored for $10 \mathrm{~min}$ and are presented with an absorbance limit of $0 \cdot 2$.

1984a). The assay conditions were chosen so that the most active uninhibited reaction never exceeded an absorbance of $1 \cdot 25$. Although the graphical output shows a limiting absorbance of $0 \cdot 2$, regressions were fitted to all readings to ensure that inhibition was assessed over the full assay period $(10 \mathrm{~min})$, regardless of $\mathrm{AChE}$ activity. Although inhibition reactions were not linear, the linear absorbance-time regressions routinely provided by the microtitre plate reader proved most robust for their characterization, since the Maximum Likelihood Program was unable to fit single- or double-exponential functions to $c a 10$ and $50 \%$ of assays, respectively.

When activity was fully inhibited (e.g. wells $5 \mathrm{~A}-\mathrm{H}$ and $12 \mathrm{~A}-\mathrm{H}$ ), the reaction rates (measured as the slope of fitted regression lines) were often negative, but typically by only -1 or $-2 \%$ relative to the uninhibited rate. This was probably due to continual slight oxidation of reduced DTNB by impurities in the Triton X-100 (Ashani \& Catravas, 1980). The brief initial activity in these 'fully inhibited' reactions could be observed by expanding the absorbance scale tenfold.

Uninhibited AChE activities, which reflect the catalytic efficiency of the enzyme, differed significantly between homozygous strains (Fig. 1, Table I). Heterozygotes, which contain equal amounts of both enzyme forms (Devonshire, 1980), had activities intermediate to the respective parental strains. These differences in uninhibited activity were taken into account by expressing each inhibited reaction rate as a percentage of AChE activity for the same insect in the absence of inhibitor; this yields an overall measure of the amount of uninhibited activity remaining during inhibition by the respective organophosphate.

Using this measure, inhibitor concentrations of $10 \mu \mathrm{M}$ dichlorvos and $0.2 \mu \mathrm{M}$ azamethiphos discriminated very effectively between the three genotypes derived from strains $S$ and WeyR, and $S$ and $49 R$, respectively, even though insensitivity factors were only 3-15-fold (Devonshire, 1987). The concentration of dichlorvos required to distinguish the 15-fold insensitivity of WeyR homozygotes from WeyR $\times S$ heterozygotes completely inhibited the AChE in S homozygotes (Fig. 2). In contrast, the much lower concentration of azamethiphos needed to detect the threefold insensitivity of $49 \mathrm{R}$ to this compound did 
TABLE I. Mean AChE activity (and standard deviation) in Musca domestica adults of six genotypes in the presence and absence of organo-phosphate inhibitors

\begin{tabular}{|c|c|c|c|c|}
\hline \multirow{2}{*}{$\begin{array}{l}\text { Strain/ } \\
\text { cross }\end{array}$} & \multirow{2}{*}{$\begin{array}{l}\text { No. of } \\
\text { flies tested }\end{array}$} & \multirow{2}{*}{$\begin{array}{l}\text { Uninhibited } \\
\text { activity }^{1}\end{array}$} & \multicolumn{2}{|c|}{$\begin{array}{c}\text { Mean } \% \text { activity remaining } \\
\text { during inhibition } b y^{2}:\end{array}$} \\
\hline & & & Dichlorvos & Azamethiphos \\
\hline $\begin{array}{l}\text { WeyR } \\
49 \mathrm{R} \\
\text { S } \\
\text { WeyR } \times 49 \mathrm{R} \\
\text { WeyR } \times \mathrm{S} \\
49 \mathrm{R} \times \mathrm{S}\end{array}$ & $\begin{array}{r}96 \\
126 \\
32 \\
95 \\
95 \\
104\end{array}$ & $\begin{array}{r}115.9(17.0) \\
45.2(7.8) \\
73.8(11.9) \\
59.9(12.1) \\
95.1(15.8) \\
70.0(17.6)\end{array}$ & $\begin{array}{r}81 \cdot 1(7 \cdot 5) \\
12 \cdot 2(5 \cdot 8) \\
-1 \cdot 8(1.5) \\
55 \cdot 5(7.9) \\
42 \cdot 4(6.9) \\
2 \cdot 7(1.6)\end{array}$ & $\begin{array}{c}0 \\
0 .(0 \cdot 3) \\
69 \cdot 1(6 \cdot 4) \\
10 \cdot 2(1.9) \\
28 \cdot 8(4.9) \\
-1.4(2.4) \\
32.7(5.2)\end{array}$ \\
\hline
\end{tabular}

Expressed as the slope of a linear regression of absorbance readings on time in the absence of inhibitors.

${ }^{2}$ Calculated as: mean inhibited activity $\times 100 /$ uninhibited activity.

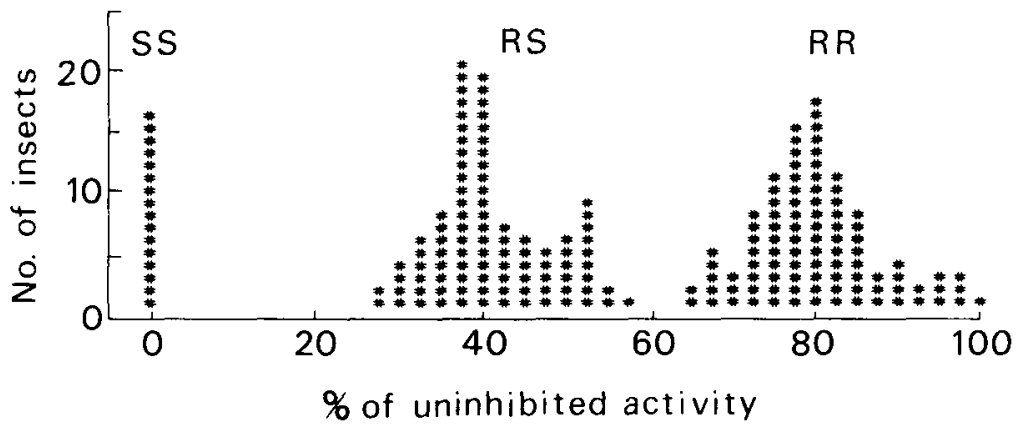

Fig. 2.-Distributions of mean percentage activity remaining during inhibition of AChE by $10 \mu \mathrm{M}$ dichlorvos for three AChE genotypes derived from strains WeyR and $\mathrm{S}$.

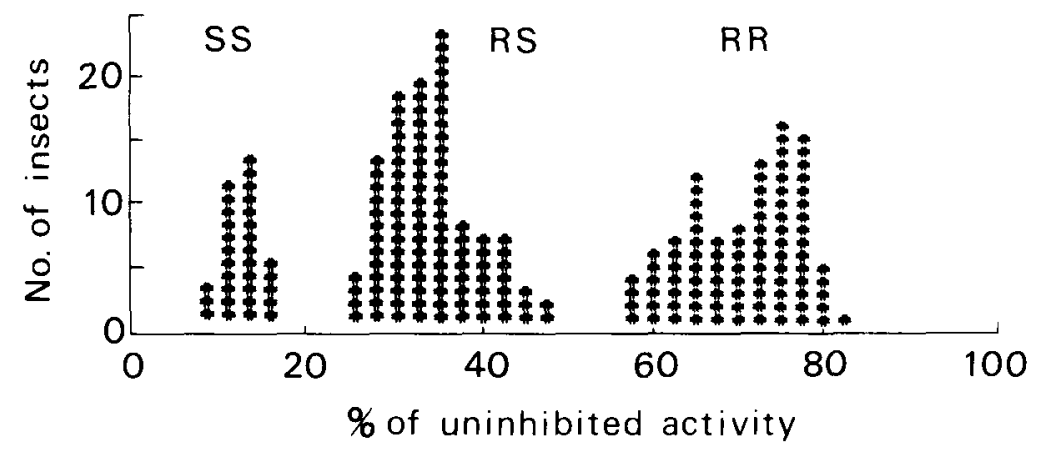

Fig. 3--Distributions of mean percentage activity remaining during inhibition of AChE by $0.2 \mu \mathrm{M}$ azamethiphos for three AChE genotypes derived from strains $49 \mathrm{R}$ and $\mathrm{S}$. 
not fully inhibit the S enzyme (Fig. 3). As in strain $\mathrm{CH}_{2}$ (Devonshire, 1987), the AChE of WeyR genotypes was very slightly hypersensitive to azamethiphos, which was consequently a useless discriminator. Similarly, the genotypes derived from 49R (Table I) were not well resolved by dichlorvos.

Differences between the three AChE variants were such that a bivariate plot of data for both inhibitors resolved fully all six possible genotypic combinations of WeyR, 49R and S, including the WeyR $\times 49 \mathrm{R}$ heterozygote (Fig. 4). Because the catalytic activities of the enzymes differed (Table I), the inhibited activity in heterozygotes was displaced towards that of the parent with the more active enzyme. Thus, the WeyR $\times S$ and WeyR $\times 49 R$ distributions are nearer to that of WeyR, and $49 R \times S$ is nearer to $S$.

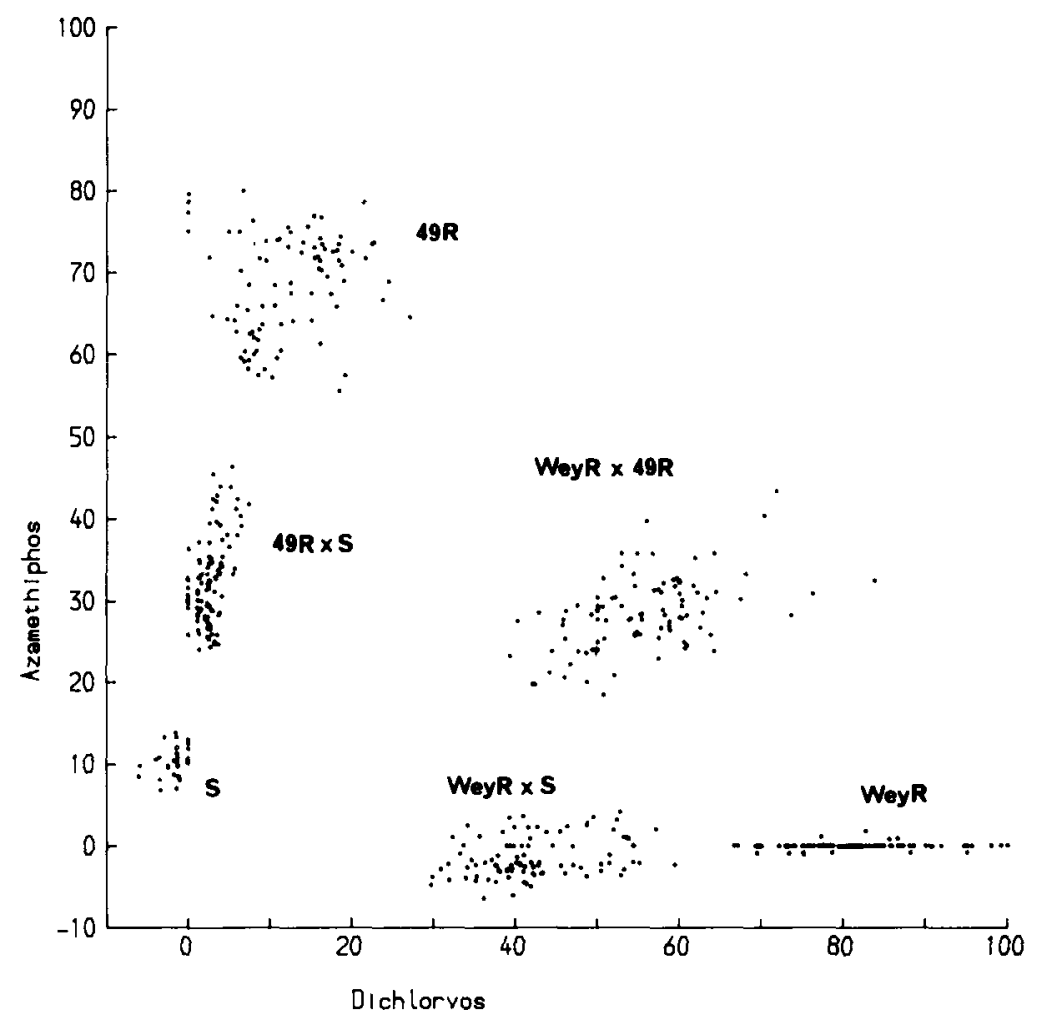

Fig. 4.-Bivariate plot of mean percentage activity remaining during inhibition of AChE by $10 \mu \mathrm{M}$ dichlorvos and $0.2 \mu \mathrm{M}$ azamethiphos for all six AChE genotypes derived from strains WeyR, $49 \mathrm{R}$ and $\mathrm{S}$.

If a kinetic microtitre plate reader is not available, end-point analysis provides a simpler, though less precise option. In this case, inhibition is assessed by a single absorbance reading at the end of the assay instead of a sequence of readings taken as the reaction proceeds. Single readings, however, cannot compensate for the varying amounts of yellow chromophores present in the insect homogenate or arising from interactions between the DTNB and endogenous thiols. This might explain why an end-point assay developed for mosquitoes (Hemingway et al., 1986) gave only limited separation of AChE genotypes, despite a 1600-fold insensitivity. Under optimal assay conditions, end-point analysis is useful for detecting insensitive AChE (Fig. 5) but will not match the resolution achieved by the continuous assay. 


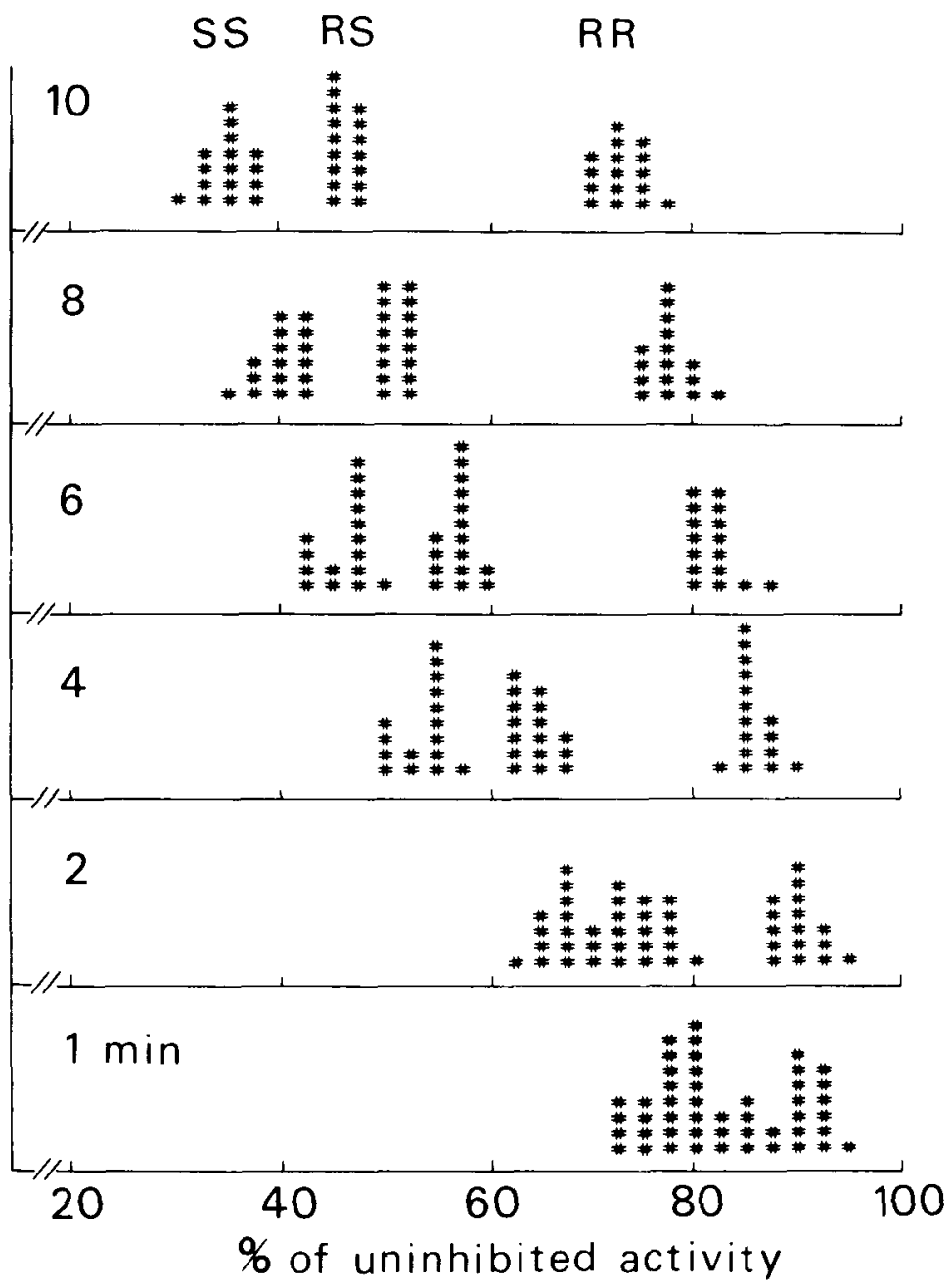

Fig. 5.-Distributions of single absorbance readings, expressed as a percentage of those in corresponding uninhibited assays, for different end-points (1$10 \mathrm{~min}$ ) during $\mathrm{AChE}$ inhibition by $0.2 \mu \mathrm{M}$ azamethiphos for three genotypes derived from strains $49 \mathrm{R}$ and $\mathrm{S}$.

Sensitive assays to monitor changes in $R$ gene frequency are a prerequisite for appraising the efficacy of proposed countermeasures to resistance in the laboratory and in the field (Sawicki \& Denholm, 1987). Those distinguishing reliably between $\mathrm{R}$ heterozygotes and homozygotes are particularly valuable in this respect, since the former are often key determinants of selection rates (Wood \& Mani, 1981; Curtis, 1985). Although assay conditions can be defined precisely for known AChE variants, they should not be used uncritically for studying field populations, which can show considerable allelic variation for AChE (Devonshire, 1987). However, since several inhibitors can be tested against the same insect, the present technique can yield an 'insesitivity profile' of individuals and strains. This should both contribute to the choice of appropriate control agents 
and provide a clearer insight into the extent of allelic variation for $\mathrm{AChE}$ in field populations.

\section{Acknowledgements}

We thank Suzanna Scott for rearing insects, Dr C. D. Kershaw for help with data analysis on the VAX, and J. Keiding (Danish Pest Infestation Control Laboratory) and Bayer AG (Leverkusen) for providing strains $49 \mathrm{r}_{2} \mathrm{~b}$ and Weymann, respectively.

\section{References}

Ashani, Y. \& Catravas, G. N. (1980). Highly reactive impurities in Triton X-100 and Brij 35: partial characterization and removal.-Analyt. Biochem. 109, 55-62.

Brown, T. M. \& Brogdon, W. G. (1987). Improved detection of insecticide resistance through conventional and molecular techniques.-A. Rev. Ent. 32, 145-162.

CurTis, C. F. (1985). Theoretical models of the use of insecticide mixtures for the management of resistance. - - Bull. ent. Res. 75, 259-265.

DeVONSHIRE, A. L. (1980). Insecticide resistance caused by decreased sensitivity of acetylcholinesterase to inhibition.- pp. 473-480 in Insect neurobiology and pesticide action (Neurotox 79).517 pp. London, Soc. Chem. Ind.

Devonshire, A. L. (1987). Biochemical studies of organophosphorus and carbamate resistance in houseflies and aphids.-pp. 239-255 in Ford, M. G., Hollomon, D. W., Khambay, B. P. S, \& Sawicki, R. M. (Eds). Combating resistance to xenobiotics: biological and chemical approaches. $-320 \mathrm{pp}$. Chichester, UK, Ellis Horwood.

Devonshire, A. L. \& Moores, G. D. (1984a). Characterisation of insecticide-insensitive acetylcholinesterase: microcomputer-based analysis of enzyme inhibition in homogenates of individual house fly (Musca domestica) heads.-Pestic. Biochem. \& Physiol. 21, 341-348.

Devonshire, A. L. \& Moores, G. D. (1984b). Different forms of insensitive acetylcholinesterase in insecticide-resistant house flies (Musca domestica).-Pestic. Biochem. \& Physiol. 21, 336-340.

Ellman, G. L., Courtney, K. D., Andres, V. \& Featherstone, R. M. (1961). A new and rapid colorimetric determination of acetylcholinesterase activity.-Biochem. Pharmac. 7, 88-95.

Farnham, A. W. (1973). Genetics of resistance of pyrethroid-selected houseflies, Musca domestica L.-Pestic. Sci. 4, 513-520.

fFrench-Constant, R. H. \& Devonshire, A. L. (1987). A multiple homogeniser for rapid sample preparation in immunoassays and electrophoresis.-Biochem. Genet. 25, 493-499.

Hama, H. (1983). Resistance to insecticides due to reduced sensitivity of acetylcholinesterase.pp. 299-331 in Georghiou, G. P. \& Saito, T. (Eds). Pest resistance to pesticides. -809 pp. New York, Plenum Press.

Hemingway, J., Smith, C., Jayawardena, K. G. I. \& Herath, P. R. J. (1986). Field and laboratory detection of the altered acetylcholinesterase resistance genes which confer organophosphate and carbamate resistance in mosquitoes (Diptera: Culicidae).-Bull. ent. Res. 76, 559-565.

Oppenoorth, F. J., Smissaert, H. R., Welling, W., van der Pas, L. J. T. \& Hitman, K. T. (1977). Insensitive acetylcholinesterase, high glutathione-S-transferase, and hydrolytic activity as resistance factors in a tetrachlorvinphos-resistant strain of house fly.-Pestic. Biochem. \& Physiol. 7, 34-47.

Raymond, M., Fournier, D., Berge, J., Cuany, A., Bride, J. M. \& Pasteur, N. (1985). Singlemosquito test to determine genotypes with an acetylcholinesterase insensitive to inhibition to propoxur insecticide.-J. Am. Mosq. Control Assoc. 1, 425-427.

Ross, G. J. S. (1987). Maximum Likelihond Program (MLP 3.08 User Manual).-Oxford, UK, Numerical Algorithms Group.

SAwICKI, R. M. (1974). Genetics of resistance of a dimethoate-selected strain of houseflies (Musca domestica $\mathrm{L}$.) to several insecticides and methylenedioxyphenyl synergists.-J. agric. Fd Chem. 22, 344-349. 
SAwicki, R. M. \& Denholm, I. (1987). Management of resistance to pesticides in cotton pests.-Trop. Pest Management 33, 262-272.

Tripathi, R. K. \& O'Brien, R. D. (1973). Insensitivity of acetylcholinesterase as a factor in resistance of houseflies to the organophosphate Rabon.-Pestic. Biochem. \& Physiol. 3, 495-498.

Wood, R. J. \& MANI, G. S. (1981). The effective dominance of resistance genes in relation to the evolution of resistance.-Pestic. Sci. 12, 573-581.

(Received 25 January 1988)

(C) C.A.B International, 1988 